\title{
Design, modeling and synthesis of an in vitro transcription rate regulatory circuit
}

\author{
Elisa Franco, Per-Ola Forsberg and Richard M. Murray
}

\begin{abstract}
This paper describes the design, modeling and realization of a synthetic in vitro circuit that aims at regulating the rate of mRNA transcription. Two DNA templates are designed to interact through their transcripts, creating negative feedback loops that will equate their transcription rates at steady state. A mathematical model is developed for this circuit, consisting of a set of ODEs derived from the mass action laws and Michaelis-Menten kinetics involving all the present chemical species. The DNA strands were accordingly designed, following thermodynamics principles and minimizing unwanted interactions. Preliminary experimental results show that the circuit is performing the expected task, by matching at steady state the transcription rates of the two DNA templates.
\end{abstract}

\section{INTRODUCTION AND BACKGROUND}

Biology is the science of this century [3]: an immense collaborative effort is today in place among different disciplines, such as physics, mathematics and engineering, aiming at the achievement of quantitative knowledge of biological processes. Building biosynthetic systems out of known components, with the same confidence as one can build a silicon device, is an extremely attractive target, still far to reach.

Trying to realize new biochemical architectures, where the user can design molecular interactions following universal engineering principles, allows not only to expand the available molecular machinery, but also to gain a better understanding of the characteristics, modularity and evolvability of existing complex molecular networks that still need to be unraveled [1].

Building a circuit out of biological components is simplified when operating in vitro: a higher control over the environment and over unwanted reactions permits to monitor more precisely the functional response of the designed system. Utilizing few components is also beneficial to the same purposes.

The topic of this paper is the design, mathematical modeling and synthesis emphin vitro of an RNA transcription rate regulatory circuit based on negative feedback. Transcription is a fundamental part of the central dogma of molecular biology and is naturally regulated in the cell: for instance it can be turned on or off by binding of transcription factors, or by secondary structure formation in the nascent RNA (see [4] and references cited therein). The dynamics of several genes

Research supported in part by the Institute for Collaborative Biotechnologies through grant DAAD19-03-D-0004 from the U.S. Army Research Office.

Elisa Franco and Richard Murray are in the Division of Engineering and Applied Sciences, California Institute of Technology, Pasadena, CA 91125. elisa,murray@cds.caltech.edu. Per-Ola Forsberg is in the Faculty of Medicine at Lund University and the Lund Institute of Technology, Lund, Sweden.per-ola.forsberg.424@student.lu.se can be coupled, and it is an interesting question whether there exist mechanisms that match the transcription rates of two or more genes.

In this work we consider a setting where two double stranded DNA (dsDNA) species are coupled through their transcripts through a mechanism of self repression: if one of the two transcripts is in excess with respect to the other, it is designed to decrease its own production by displacing a single stranded DNA (ssDNA) molecule that completes the template promoter region. Thanks to this negative feedback loop which is a switch-off type of functionality, at equilibrium the two transcription rates are equal. Only two enzyme species are utilized for the realization of this circuit.

The first in vitro transcriptional switches were designed and realized by Kim [7], [6] as a possible biological implementation of neural networks. More complex cell-free environments for quantitative analysis have been proposed in [8], where protein signaling patters are considered. However, the computational power of a simple setting comprising only ssDNA, dsDNA (or RNA) and few enzymes has been theoretically proven to be superior [5] by virtue of its simplicity. The same thermodynamics principles utilized to realize transcriptional switches are useful to realize several other systems presenting a circuit-like behavior [9] or even to create nanomolecular devices [2]. A further motivation in focusing our attention on nucleic acids lies in their important role in the control of gene expression, which is being acknowledged and studied with increasing interest [4].

The main contribution of this paper is that of presenting a new architecture based on transcriptional switches that realizes a regulatory mechanism never considered before. Following fundamental engineering principles, a negative feedback rate regulator (NFRR) circuit was designed and mathematically modeled starting from the occurring biochemical reactions; the system was then synthesized and tested. The employed pool of biological machinery is of interest because it can be used to realize a variety of molecular devices with different functionalities, despite its simplicity and low number of components. Preliminary experimental results presented in this paper show that the NFRR can regulate the transcription process in the correct way. Further improvement of the strands design is needed in order to refine such results.

The paper is organized as follows: Section II describes in further depth the design, modeling and synthesis of the rate regulatory circuit. Section III presents some preliminary experimental results. Finally, Section IV summarizes the obtained results and discusses the future directions of this 
research

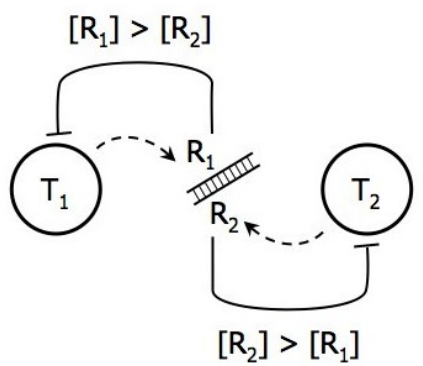

Fig. 1. Scheme for the NFRR. The dsDNA templates $T_{1}$ and $T_{2}$ produce transcripts that generate a double stranded complex $R_{1} R_{2}$. If the RNA species $R_{1}$ is in excess, it will inhibit its own production; if $R_{2}$ is in excess, it inhibits its own production.

\section{CiRCUit DESCRIPTION, MODELING AND SYNTHESIS}

\section{A. Circuit design}

In this work, the problem of how to equate the transcription rates of two synthetic RNA products is specifically considered. A simple negative feedback based in vitro system is taken into account, which is composed of DNA and RNA molecules and two enzyme species [6]. Two dsDNA templates $T_{1}, T_{2}$ are incomplete in their promoter region: two ssDNA molecules called activators $A_{1}, A_{2}$ can bind the templates completing the promoter and allowing the enzyme RNA polymerase $\left(\mathbf{R}_{\mathbf{p}}\right)$ to operate the transcription of RNA species $R_{1}, R_{2}$. The two RNAs of interest are designed to bind, forming a double stranded complex potentially available for further processing. By construction, if either of the two products is in excess with respect to the other which means its transcription rate is higher - it will inhibit its own production by displacing the DNA activator from the DNA template. Since both transcripts have this selfrepression function, at steady state their production rates will equate. RNase $\mathrm{H}\left(\mathbf{R}_{\mathbf{h}}\right)$, the other enzyme species present, allows degradation of DNA-RNA hybrids introducing a further level of dynamic adaptation. The NFRR architecture is schematically described in Figure 1.

Considering an in vitro setting, the utilization of DNA template strands with incomplete promoter regions [7], [6] easily allows to synthesize a negative feedback loop using few components. Transcription is inhibited by default, but can be restored by addition of a ssDNA activator that completes the promoter. The mechanism allowing to turn off the template is known as branch migration: if the activator strand is provided with a toehold region [10], it can be displaced by specifically designed RNA or DNA molecules, the inhibitors. This toehold region is a $7-10$ nucleotides long overhang that remains exposed when the activator is bound to the template: inhibitors have sequences complementary to the whole activator strand, and the molecular complex inhibitor/activator is formed more favorably than the template/activator one, with a gain of free energy. For instance, $A_{1}$ can bind to $T_{1}$, with free energy $-4.289437 \mathrm{kcal} / \mathrm{mol}$, while the complex $R_{1} A_{1}$ presents a free energy of $-6.374918 \mathrm{kcal} / \mathrm{mol}$ and is therefore a more favorable reaction. The very transcript of the template can be designed to serve that function: the circuit will in such case be a self-inhibitor. Figure 2 shows the sequence structure for template $T_{i}$ : the different regulatory regions are labeled and highlighted in different colors. The hairpin region at the $3^{\prime}$ end of the template (and therefore of the transcript) is required in order to prevent the RNA polymerase from extending the transcript creating spurious RNA [7], Ch. 3.

$\mathrm{Ri}$
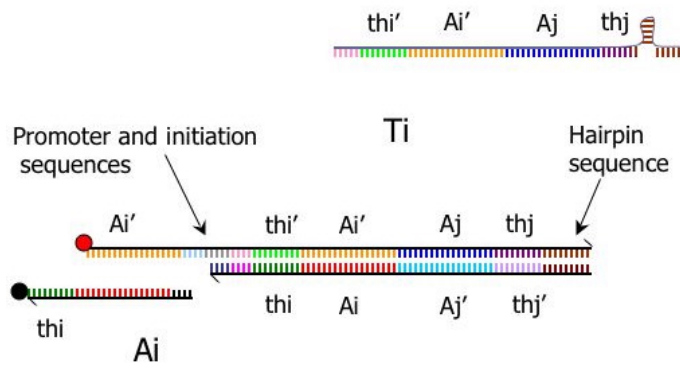

Fig. 2. Structure of template $T_{i}$. The arrow tick at the end of the strands indicates the $5^{\prime}$ to $3^{\prime}$ direction. Starting from the 5' (left): fluorophore ${ }_{i}$ (red circle), complementary $A_{i}$ region (yellow, 23 nucleotides), promoter region and initiation sequences (cyan, grey and pink, 28 nucleotides), complementary toehold $i$ region (green, 8 nucleotides), complementary $A_{i}$ region (orange, 22 nucleotides), $A_{j}$ region (dark blue, 22 nucleotides), toehold ${ }_{j}$ region (purple, 8 nucleotides) and at the 3 ' end hairpin region (brown, 16 nucleotides). The sequence of the transcript $R_{i}$ comprises all the regions of $T_{i}$ right after the promoter. Starting from the 3' end (left) for $A_{i}$ : Quencher (black circle), toehold ${ }_{i}$ region (turquoise, 8 nucleotides), activator $A_{i}$ region (orange, 22 nucleotides) and the missing part of the promoter (dark grey, 5 nucleotides).

The complete set of reactions is shown in Figure 3. To fulfill the desired constraints, the design of the transcripts is such that binding might occur also between $T_{i}$ and $R_{j}$, which is considered a further off state.

\section{B. Mathematical modeling}

A model of the NFRR can be derived from the chemical reactions occurring in the system. All three possible states of the template will be considered: the on state where activator and template are bound and form the complex $T_{i} A_{i}$; the off states given by free $T_{i}$ and by $R_{j}$ bound to $T_{i}$ forming $T_{i} R_{j}$. An off state still allows for $\mathbf{R}_{\mathbf{p}}$ weak binding and transcription. Throughout this derivation, the dissociation constants are omitted when assumed to be negligible. It is hypothesized that the concentration of enzymes is considerably lower than that of the DNA molecules, allowing the classical steady state assumption for Michaelis-Menten kinetics. 


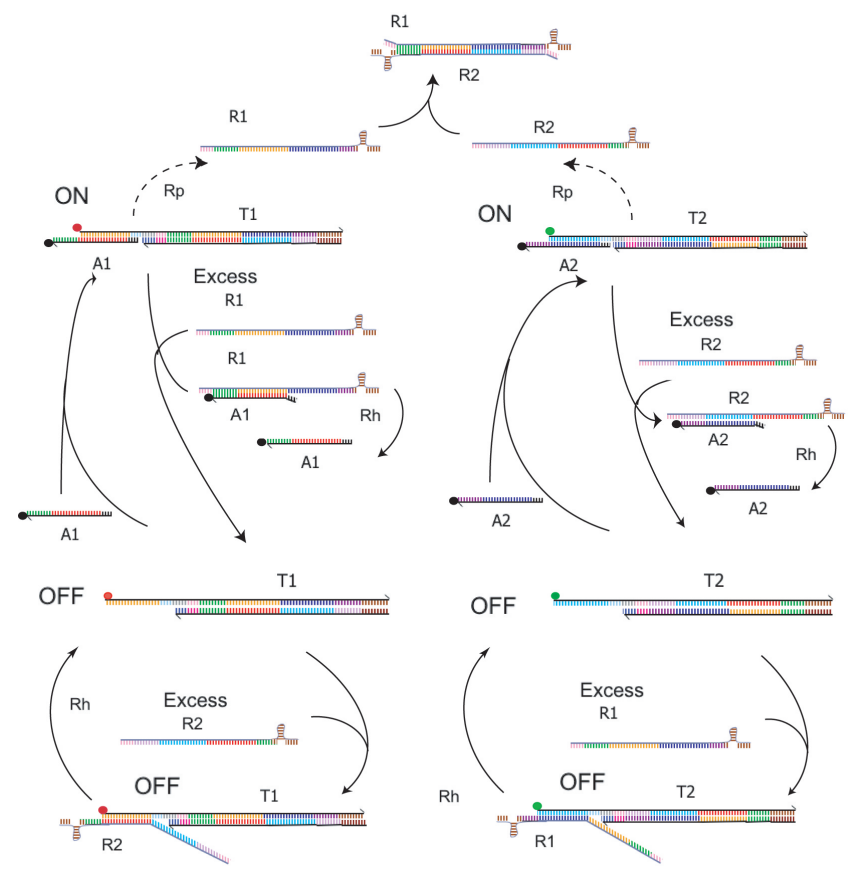

Fig. 3. The NFRR consists of templates $T_{1}, T_{2}$ with incomplete promoter regions and activators $A_{1}, A_{2}$ which complete the missing part of the promoter. Binding of $T_{i}$ to $A_{i}$, turning the sub-circuit into the on state, starts transcription of RNA strand $R_{i}$. RNA strands bind and form the $R_{i} R_{j}$ complex. If either $R_{i}$ is in excess, it will strip off $A_{i}$ from $T_{i}$. Also, if $A_{i}$ is not bound to $T_{i}, R_{j}$ in excess will bind to $T_{i}$, effectively turning the template off.

The mass action reactions are, for $i \in\{1,2\}, j \in\{2,1\}$ :

$$
\begin{gathered}
T_{i}+A_{i} \stackrel{k_{T_{i} A_{i}}}{\rightarrow} T_{i} A_{i} \\
R_{i}+A_{i} \stackrel{k_{R_{i} A_{i}}}{\rightarrow} R_{i} A_{i} \\
R_{i}+T_{i} A_{i} \stackrel{k_{R_{i} T_{i} A_{i}}}{\rightarrow} R_{i} A_{i}+T_{i} \\
R_{i}+R_{j} \stackrel{k_{R_{i} R_{j}}}{\rightarrow} R_{i} R_{j} \\
R_{j}+T_{i} \stackrel{k_{R_{j} T_{i}}}{\rightarrow} R_{j} T_{i}
\end{gathered}
$$

The enzymatic reactions are, for $i \in\{1,2\}, j \in\{2,1\}$ :

$$
\begin{aligned}
& \mathbf{R}_{\mathbf{p}}+T_{i} A_{i} \underset{k_{O N i i}^{-}}{\stackrel{k_{\text {ONii }}^{+}}{\rightleftarrows}} \mathbf{R}_{\mathbf{p}} \cdot T_{i} A_{i} \stackrel{k_{\text {cat } O N i i}}{\longrightarrow} \mathbf{R}_{\mathbf{p}}+T_{i} A_{i}+R_{i} \\
& \mathbf{R}_{\mathbf{p}}+T_{i} \underset{k_{O F F i i}^{-}}{\stackrel{k_{\text {FF }}^{+}}{\rightleftarrows}} \mathbf{R}_{\mathbf{p}} \cdot T_{i} \stackrel{k_{\text {cat } O F F i i}}{\rightarrow} \mathbf{R}_{\mathbf{p}}+T_{i}+R_{i} \\
& \mathbf{R}_{\mathbf{h}}+R_{i} A_{i} \underset{k_{H i i}^{-}}{\stackrel{k_{H i i}^{+}}{\rightleftarrows}} \mathbf{R}_{\mathbf{h}} \cdot R_{i} A_{i} \stackrel{k_{c a t H i i}}{\longrightarrow} \mathbf{R}_{\mathbf{h}}+A_{i} \\
& \mathbf{R}_{\mathbf{h}}+R_{j} T_{i} \underset{k_{H j i}^{-}}{\stackrel{k_{H j i}^{+}}{\rightleftarrows}} \mathbf{R}_{\mathbf{h}} \cdot R_{j} T_{i} \stackrel{k_{c a t H j i}}{\longrightarrow} \mathbf{R}_{\mathbf{h}}+T_{i} \\
& \mathbf{R}_{\mathbf{p}}+R_{j} T_{i} \underset{k_{O F F j i}^{-}}{\stackrel{k_{O F F j i}^{+}}{\rightleftarrows}} \mathbf{R}_{\mathbf{p}} \cdot R_{j} T_{i} \stackrel{k_{\text {catoF }} \stackrel{ }{\rightarrow}}{\rightarrow} \mathbf{R}_{\mathbf{p}}+R_{j} T_{i}+R_{i}
\end{aligned}
$$

as follows:

$$
\begin{aligned}
\frac{d}{d t}\left[T_{i}\right]= & -k_{T_{i} A_{i}}\left[T_{i}\right]\left[A_{i}\right]+k_{R_{i} T_{i} A_{i}}\left[R_{i}\right]\left[T_{i} A_{i}\right]- \\
& -k_{R_{j} T_{i}}\left[R_{j}\right]\left[T_{i}\right]+k_{\text {cat } H i}\left[\mathbf{R}_{\mathbf{h}} \cdot R_{j} T_{i}\right] \\
\frac{d}{d t}\left[A_{i}\right]= & -k_{T_{i} A_{i}}\left[T_{i}\right]\left[A_{i}\right]-k_{R_{i} A_{i}}\left[R_{i}\right]\left[A_{i}\right]+ \\
& +k_{\text {cat Hii }}\left[\mathbf{R}_{\mathbf{h}} \cdot R_{i} A_{i}\right] \\
\frac{d}{d t}\left[R_{i}\right]= & -k_{R_{i} R_{j}}\left[R_{i}\right]\left[R_{j}\right]-k_{R_{i} T_{i} A_{i}}\left[R_{i}\right]\left[T_{i} A_{i}\right]- \\
& -k_{R_{i} T_{j}}\left[R_{i}\right]\left[T_{j}\right]-k_{R_{i} A_{i}}\left[R_{i}\right]\left[A_{i}\right]+ \\
& +k_{\text {catONii }}\left[\mathbf{R}_{\mathbf{p}} \cdot T_{i} A_{i}\right]+k_{c a t O F F i i}\left[\mathbf{R}_{\mathbf{p}} \cdot T_{i}\right]+ \\
& +k_{\text {catOFFji }}\left[\mathbf{R}_{\mathbf{p}} \cdot R_{j} T_{i}\right] \\
\frac{d}{d t}\left[R_{i} R_{j}\right]= & +k_{R_{i} R_{j}}\left[R_{i}\right]\left[R_{j}\right] \\
\frac{d}{d t}\left[R_{j} T_{i}\right]= & +k_{R_{j} T_{i}}\left[R_{j}\right]\left[T_{i}\right]-k_{c a t H i j}\left[\mathbf{R}_{\mathbf{h}} \cdot R_{j} T_{i}\right]
\end{aligned}
$$

A further equation can be derived for the rate of production of each RNA species $R_{i}$. The molecular complexes that appear in the right hand side of the above equation can be expressed as a function of the states with some standard steps. Mass conservation immediately yields $\left[T_{i} A_{i}\right]=$ $\left[T_{i}^{\text {tot }}\right]-\left[T_{i}\right]-\left[R_{j} T_{i}\right]$ and $\left[R_{i} A_{i}\right]=\left[A_{i}^{\text {tot }}\right]-\left[A_{i}\right]-\left[T_{i} A_{i}\right]$.

Furthermore, assuming that binding of the enzyme is faster than transcription or degradation in equation (2) and defining the Michaelis-Menten coefficients, e.g. for the ON state of the template $k_{M O N i i}=\frac{k_{-O N i i}+k_{\text {catONii }}}{k_{+O N i i}}$, it is possible to use mass conservation laws to obtain the following expressions involving the enzyme free concentrations:

$$
\begin{aligned}
& {\left[\mathbf{R}_{\mathbf{p}}{ }^{t o t}\right] }=\left[\mathbf{R}_{\mathbf{p}}\right]\left(1+\frac{\left[T_{1} A_{1}\right]}{k_{M O N 11}}+\frac{\left[T_{1}\right]}{k_{M O F F 11}}+\right. \\
&\left.\frac{\left[T_{2} A_{2}\right]}{k_{M O N 22}}+\frac{\left[T_{2}\right]}{K_{M O F F 22}}+\frac{\left[R_{2} T_{1}\right]}{k_{M O F F 21}}+\frac{\left[R_{1} T_{2}\right]}{k_{M O F F 12}}\right) \\
& {\left[\mathbf{R}_{\mathbf{h}}{ }^{t o t}\right]=\left[\mathbf{R}_{\mathbf{p}}\right]\left(1+\frac{\left[R_{1} A_{1}\right]}{k_{M H 11}}+\frac{\left[R_{2} A_{2}\right]}{k_{M H 22}}+\frac{\left[R_{2} T_{1}\right]}{k_{M H 21}}+\frac{\left[R_{1} T_{2}\right]}{k_{M H 12}}\right) }
\end{aligned}
$$

We can easily rewrite the above equations as $\left[\mathbf{R}_{\mathbf{p}}\right]=$ $\frac{\left[\mathbf{R}_{\mathbf{p}}{ }^{t o t}\right]}{P}$ and $\left[\mathbf{R}_{\mathbf{h}}\right]=\frac{\left[\mathbf{R}_{\mathbf{h}}{ }^{t o t}\right]}{H}$, with a straightforward definition of the coefficients $P$ and $H$. The remaining expressions to be utilized within equation (3) can be then obtained:

$$
\begin{aligned}
{\left[\mathbf{R}_{\mathbf{p}} \cdot T_{i} A_{i}\right] } & =\frac{\left[\mathbf{R}_{\mathbf{p}}{ }^{t o t}\right]\left[T_{i} A_{i}\right]}{P \cdot k_{M O N i i}} \\
{\left[\mathbf{R}_{\mathbf{p}} \cdot R_{j} T_{i}\right] } & =\frac{\left[\mathbf{R}_{\mathbf{p}}{ }^{t o t}\right]\left[R_{j} T_{i}\right]}{P \cdot k_{M O F F j i}} \\
{\left[\mathbf{R}_{\mathbf{p}} \cdot T_{i}\right] } & =\frac{\left[\mathbf{R}_{\mathbf{p}}{ }^{t o t}\right]\left[T_{i}\right]}{P \cdot k_{M O F F i i}} \\
{\left[\mathbf{R}_{\mathbf{h}} \cdot R_{i} A_{i}\right] } & =\frac{\left[\mathbf{R}_{\mathbf{h}}{ }^{t o t}\right]\left[R_{i} A_{i}\right]}{H \cdot k_{M H i i}} \\
{\left[\mathbf{R}_{\mathbf{h}} \cdot R_{j} T_{i}\right] } & =\frac{\left[\mathbf{R}_{\mathbf{h}}{ }^{t o t}\right]\left[R_{j} T_{i}\right]}{H \cdot k_{M H j i}}
\end{aligned}
$$

Given (1) and (2) it is straightforward to derive a set of ODEs 
The nonlinear set of equations (3) was numerically analyzed using the MATLAB ode23s solver. The parameter values used in these simulations are reported in Table I. Such parameters were taken from [6], where a bistable switch was synthesized. Our system presents indeed the same type of reactions: the length and composition of the DNA strands are analogous and the enzymatic reactions are essentially the same. The parameters are chosen so that the two sub-circuits are identical: this is a simplifying assumption that helps to gain intuition on the performance of the NFRR by just creating an imbalance in the concentration of the strands. In particular, utilizing the parameters listed in Table I, and initial conditions $T_{1}^{\text {tot }}=600 \mathrm{nM}, T_{2}^{\text {tot }}=300 \mathrm{nM}, A_{1}^{\text {tot }}=600 \mathrm{nM}$, $A_{2}^{t o t}=300 n \mathbf{M}, \mathbf{R}_{\mathbf{p}}{ }^{\text {tot }}=20 n \mathbf{M}$ and $\mathbf{R}_{\mathbf{h}}{ }^{t o t}=3 n \mathbf{M}$ the system dynamics are shown in Figure 4. The free amount of $T_{1}$ and $T_{2}$ corresponds to the amount of template that is in an off state
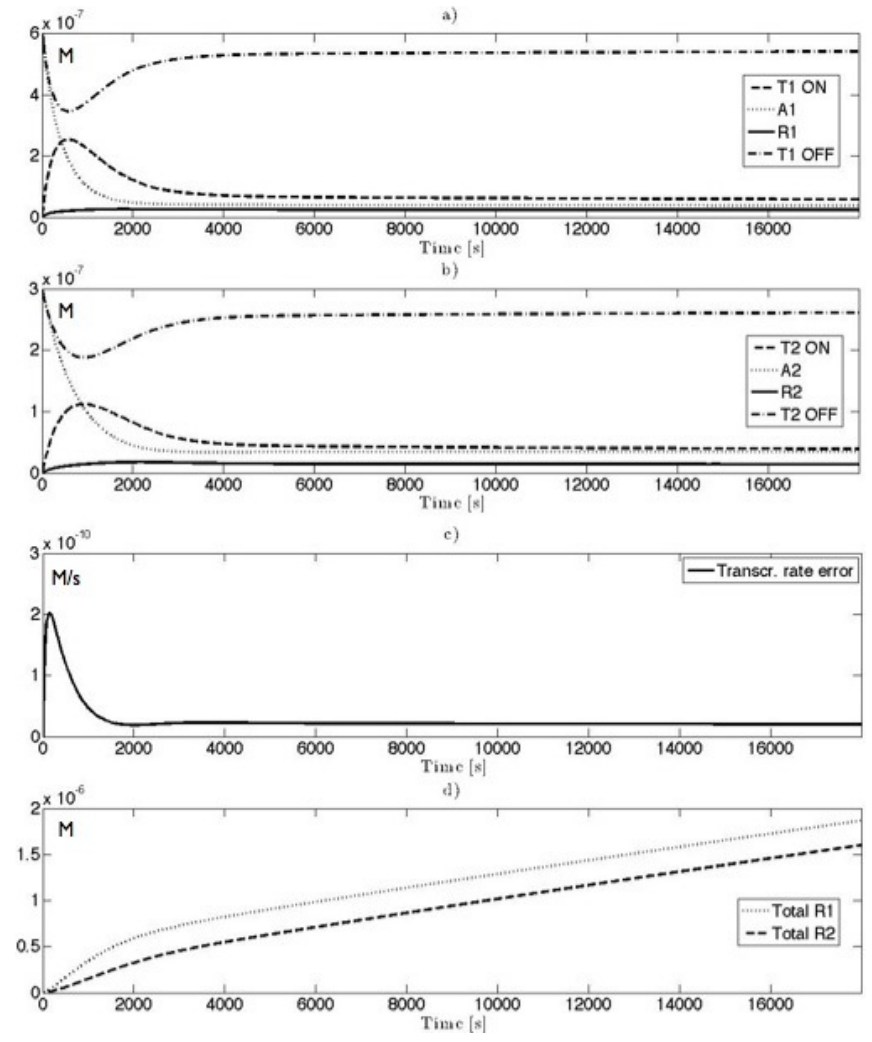

Fig. 4. a) Concentrations time profiles for sub-circuit 1 b) Concentrations time profiles for sub-circuit $2 \mathrm{c}$ ) Time profile of the transcription rate error, defined as $\frac{d}{d t} R_{1}-\frac{d}{d t} R_{2}$ d) Time profile of the total amount of RNA produced by the two sub-circuits. The curves become parallel under the feedback loop action.

\section{Circuit synthesis}

The DNA strands were designed by thermodynamic analysis using Nupack, a software package developed at Caltech and available online at http://www.nupack.org. The sequences, whose length varies from 35 nucleotides (activators) to 126 nucleotides (templates), are synthetic and do not represent existing genetic information; they were optimized so that the free energy would be in the correct
TABLE I

SIMULATION PARAMETERS FOR EQUATIONS (3)

\begin{tabular}{|l|l|l|}
\hline Units: $[s / M]$ & Units: $[1 / s]$ & Units: $[M]$ \\
\hline$k_{T_{i} A_{i}}=4 \cdot 10^{3}$ & $k_{c a t O N_{i i}}=0.064$ & $k_{M O N_{i i}}=250 \cdot 10^{-9}$ \\
\hline$k_{T_{i} A_{i} R_{i}}=5 \cdot 10^{4}$ & $k_{c a t O F F_{i i}}=1 \cdot 10^{-3}$ & $k_{M O F F_{i}}=1 \cdot 10^{-6}$ \\
\hline$k_{A_{i} R_{i}}=5 \cdot 10^{4}$ & $k_{c a t O F F_{i j}}=1 \cdot 10^{-3}$ & $k_{M O F F_{i j}}=1 \cdot 10^{-6}$ \\
\hline$k_{R_{i} T_{j}}=1 \cdot 10^{3}$ & $k_{c a t H_{i i}}=.106$ & $k_{M H_{i i}}=50 \cdot 10^{-9}$ \\
\hline$k_{R_{i} R_{j}}=2 \cdot 10^{5}$ & $k_{c a t H_{j i}}=.106$ & $k_{M H_{j i}}=50 \cdot 10^{-9}$ \\
\hline
\end{tabular}

range to yield the desired reactions, and to avoid unwanted secondary structures and crosstalk. Further constraints on the length and structure of the strands, which can affect the transcription efficiency and fidelity, were taken into account referring to [7], Chapter 3.4. The promoter used for both templates is a $T 7$ promoter. The strands were purchased from Integrated DNA Technologies (http://www.idtdna.com) and modified to include fluorophores at the 5 ' end of the templates and quenchers at the 3' end of the activators. Fluorophores are molecules that absorb light at a specific wavelength and emit light at a specific different wavelength; quenchers are molecules that only absorb light without emitting, and no emission is detected when they are in proximity of a fluorophore. Specifically, the brand name of the fluorophore attached to $T_{1}$ is TYE563, whose absorptionemission spectrum has peaks at $549 \mathrm{~nm}$ and $563 \mathrm{~nm}$ respectively; the fluorophore added to $T_{2}$ is TYE665, $647 n \mathrm{~m}-$ $665 \mathrm{~nm}$. The quencher utilized to mark the activators is IOWA black RQ, from the same vendor. Labeling the strands with fluorophores and quenchers allows to monitor the on (activator bound, fluorescence quenched) or off (activator unbound, fluorescence signal present) state of the templates by measuring fluorescence over time. Figure 3 shows all the reactions; the different regions of the strands are highlighted in different colors. The enzymes used have been purchased by Ambion (T7 RNA polymerase, E. coli cloned RNAse H), together with the nucleotides and buffer (T7 Shortmegascript kit).

\section{RESUlts}

A spectrofluorimeter (Horiba Fluorolog) was utilized to monitor the fluorescence $\Phi(t)$ of the two templates over time. All experiments are run at a temperature of $37^{\circ} \mathrm{C}$ to maximize the enzymes activity. A typical experiment consists of three stages: measuring the basal fluorescence of the templates in solution $\Phi_{\max }$, without the quenchers; measuring the minimum fluorescence $\Phi_{\text {min }}$, when adding the activators that will bind to the corresponding templates, quenching their fluorophores; finally, addition of enzymes starts transcription of the RNA products. After data are collected, they are normalized with respect to $\Phi_{\min }$ and $\Phi_{\max }$, and are then expressed in normalized units:

$$
\Phi_{N}(t)=\frac{\Phi_{\max }-\Phi(t)}{\Phi_{\max }-\Phi_{\min }}
$$

Figure 5 shows the experimental results for $T_{1}$ and $T_{2}$ separately considered, at a concentration of $300 n \mathrm{M}$; the 
concentration of their activators is also $300 n \mathrm{M}$. The analysis of the NFRR mathematical model showed worse performance with an excess of activator strands, therefore in our experiments the concentration of the templates equals that of the activators. Enzymes are added with a target volume (vendors do not provide enzymatic concentrations): RNA polymerase was added as $7 \%$ of the total volume (i.e. $4.9 \mu \mathrm{L}$ for a total volume of $70 \mu \mathrm{L}$ ), while RNase $\mathrm{H}$ is added as $11 \%$ of the RNA polymerase volume. The same enzyme volume percentage has been used throughout all the presented experimental results.

According to the measured signal, the percentage of strand that is turned off with this activator amount falls around $85 \%$ for $T_{1}$ and $65 \%$ for $T_{2}$, with respect to the basal fluorescence. This may be caused by different reasons: a transcription rate lower than expected, due to the increased length of the transcript, would drive the system to that type of equilibrium. Dilution effects need also to be taken into account, as after addition of enzyme the solution volume increases by about $14 \%$ and therefore the initial strand concentrations drop accordingly. The drop in the fluorescence signal was preliminarly tested and ranges between 10-12\% when there is a $14 \%$ increase in volume; further investigation needs to be done in this sense to reveal nonlinearities in the fluorescence decay at different template concentrations.

It should be noted in Figure $5 \mathrm{~b}$ ) that the rate at which template $T_{2}$ turns off is considerably slower than that of template $T_{1}$, Figure 5 a). This might be due to a hairpin structure that is present in the activator, whose effect was considered negligible at the design stage. The fluorophore utilized for $T_{2}$ (which had not been previously characterized in our laboratory) may also slow down the self inhibitory pathway.

Figures 6 to 9 are ratio plots showing the experimental and simulated results for steady state analysis of the circuit. The ratio of the templates on at the initial condition is plotted versus the ratio of the templates on at steady state: ideally, at steady state such ratio should be close to one. In the experiments, a ratio plot $T_{2} / T_{1}$ signifies that the concentration of $T_{1}$ was fixed at $300 \mathrm{nM}$ while the concentration of $T_{2}$ was varied in the range $100-600 n \mathrm{M}$; vice versa for the ratio plot $T_{1} / T_{2}$.

The model behavior is followed by the model described in equations (3), with the parameters given in Table I (solid line in Figures 6 to 9). In the absence of feedback loops, and assuming that the two systems are balanced, the ratio plot becomes a straight line of slope one (dotted line). The relative error of the model with respect to the experimental data (dashed line in Figures 6 to 9) is up to $50 \%$. The considerations done before regarding the dilution effects and the lower self inhibition rate for $T_{2}$ can be used to better interpret the data.

If the self inhibitory pathway for $T_{2}$ is one order of magnitude slower, then the model behavior (dashed-dotted line) becomes slightly closer to that experimentally measured in the lower template ratio range.

The data can be alternatively adjusted taking into account dilution effects, by assuming that the maximum off steady states are those tested in the separate experiments, shown in Figure 5. Specifically, the adjusted data can be premultiplied by factors $C_{\Phi 1} \triangleq\left(1-\bar{\Phi}_{T_{1}} /\left[T_{1}\right](0)\right)$ and $C_{\Phi 2} \triangleq$ $\left(1-\bar{\Phi}_{T_{2}} /\left[T_{2}\right](0)\right)$, where $\bar{\Phi}_{T_{i}}$ are the normalized off steady state average fluorescence signals measured for each template considered separately (see Figure 5). Figures 8 and 9 show the adjusted data (dashed-dotted line) together with the raw ones and the ratio plot predicted using the parameters in Table I.

The current analysis shows that the slower self inhibition for sub-circuit 2 is a better fit for the gathered data. Most likely though, a combination of this effect together with dilution would explain the difference between the collected data and the idealized model.

Further quantitative analysis is needed for the NFRR: in particular, polyacrylamide gel electrophoresis will be used to verify the production rates of the strands, separately and coupled. The strands need to be redesigned to this aim, since currently there is no significant length difference in the transcripts that would allow separation in a gel analysis.
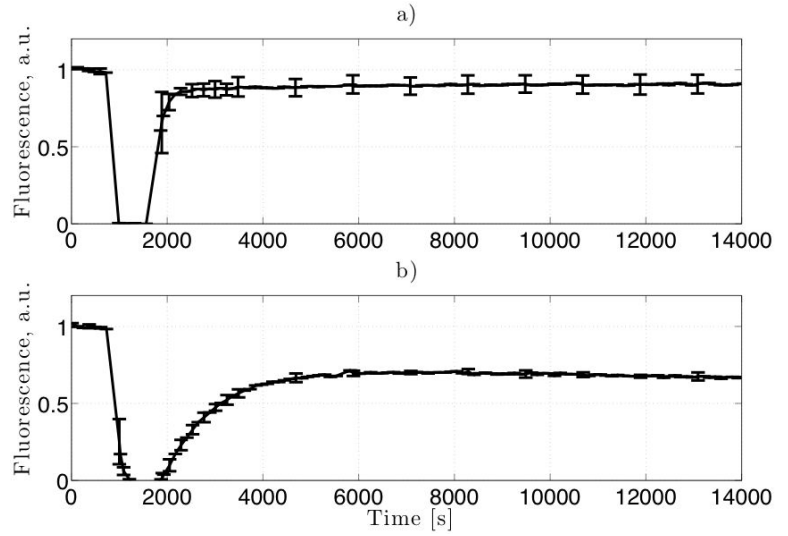

Fig. 5. a) Fluorescence of template $T_{1}$ b) Fluorescence of template $T_{2}$. Errorbars are shown each two datapoints for the normalization stage, each 10 datapoints for the steady state regime.

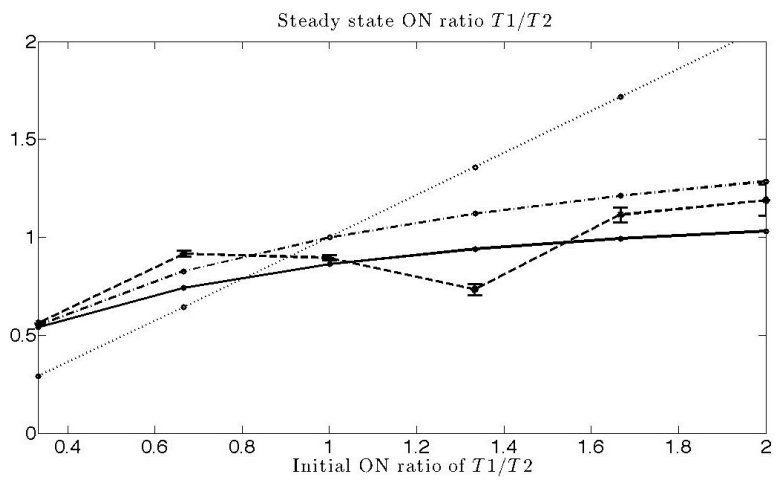

Fig. 6. Initial versus steady state ratio of $T_{1}$ over $T_{2}$ ON. Legend: - simulated behavior of the system, : simulated behavior in absence of negative feedback loops, - - simulated behavior assuming lower transcription rates, -- experimental results. 


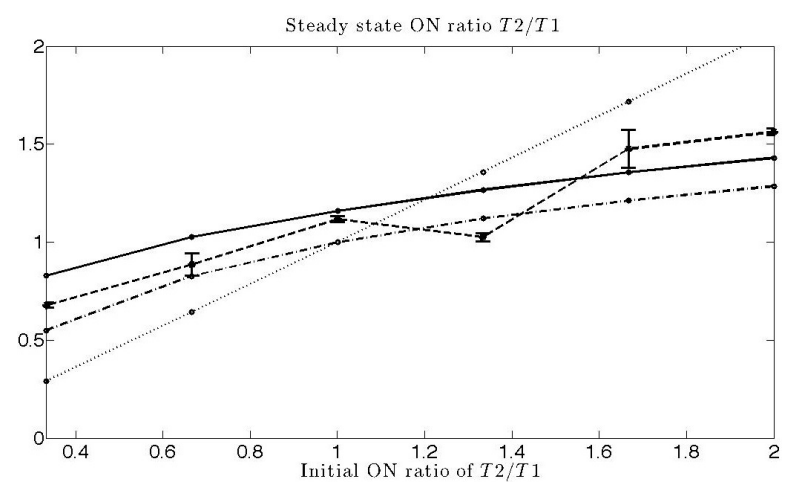

Fig. 7. Initial versus steady state ratio of $T_{2}$ over $T_{1}$ ON. Legend: - simulated behavior of the system, : simulated behavior in absence of negative feedback loops, $\cdot-$ simulated behavior assuming lower transcription rates, -- experimental results.

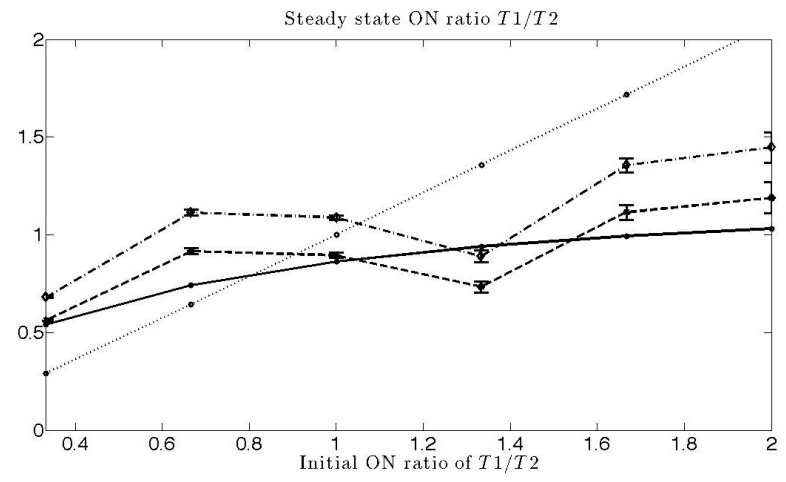

Fig. 8. Initial versus steady state ratio of $T_{1}$ over $T_{2}$ ON. Legend: - simulated behavior of the system, : simulated behavior in absence of negative feedback loops, -- experimental results, $\cdot-$ experimental results taking into account dilution effects.

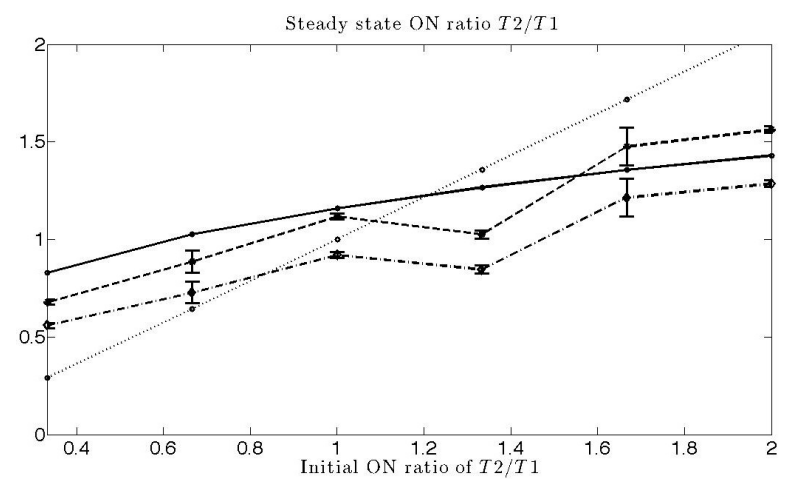

Fig. 9. Initial versus steady state ratio of $T_{2}$ over $T_{1}$ ON. Legend: - simulated behavior of the system, : simulated behavior in absence of negative feedback loops, -- experimental results, $\cdot-$ experimental results taking into account dilution effects.

\section{CONCLUSION AND Future Work}

A new biosynthetic circuit performing transcription rate regulation between two DNA templates has been presented in this paper. This regulatory system has been modeled, synthesized and tested: the preliminary experimental results show that the desired behavior is achieved to a certain extent. Experimental issues that need to be explained are the different off-switch rates of the two sub-circuits, and their inability to completely turn OFF when the activator amount equals the corresponding template concentration. In order to solve these issues, the sequences will be redesigned; this will allow further quantitative analysis through gel electrophoresis.

The rate regulation feature can in principle be obtained by designing the two strands for cross-activation instead of self-inhibition, as sketched in Figure 10. The mathematical analysis of this alternative design and its experimental testing are currently being considered.

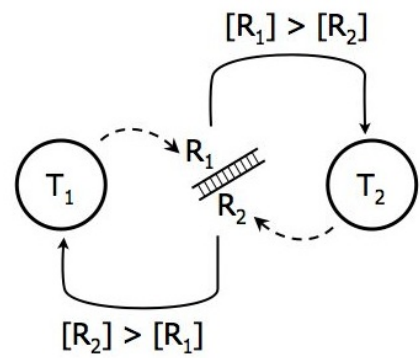

Fig. 10. Scheme for the positive feedback regulatory circuit. The DNA templates $T_{1}$ and $T_{2}$ produce transcripts that generate a double stranded complex $R_{1} R_{2}$. If the RNA species $R_{1}$ is in excess, it promotes the production of $R_{2}$; if $R_{2}$ is in excess, it promotes the production of $R_{1}$.

Acknowledgments All the experiments presented in this paper were done in Erik Winfree's laboratory. The authors would like to thank Erik Winfree, Jongmin Kim and all the members of the DNA group at Caltech for their helpful advise during the development of this project.

\section{REFERENCES}

[1] Alon, U. An Introduction to Systems Biology: Design Principles of Biological Circuits. Chapman \& Hall/CRC, 2006

[2] Bishop, J. and Klavins, E. An Improved Autonomous DNA Nanomotor. Nano Letters, 2007, 9, 2574-7

[3] Dyson, F. Our Biotech Future. New York Review of Books, July 19, 2007. Available online: http://www.nybooks.com/articles/20370.

[4] Isaacs, F. J., Dwyer, D. J. and Collins, J. J. RNA synthetic biology. Nature Biotechnology, 2006, 24, 545-554

[5] Kim, J., Hopfield J. J. and Winfree, E. Neural Network Computation by in vitro Transcriptional Circuits. Advances in Neural Information Processing Systems (NIPS), 2004, 17, 681-688.

[6] Kim, J., White, K. S. and Winfree, E. Construction of an In Vitro Bistable Circuit from Synthetic Transcriptional Switches. Molecular Systems Biology, 2006, 68

[7] Kim, J. In Vitro Synthetic Transcriptional Networks. California Institute of Technology, 2006

[8] Noireaux, V., Bar-Ziv, R. and Libchaber, A. Principles of cell-free genetic circuit assembly. Proceedings Of The National Academy Of Sciences Of The United States Of America, 2003, 100, 12672-12677

[9] Seelig, G., Soloveichik, D., Zhang, D. Y. and Winfree, E. Enzyme-free nucleic acid logic circuits. Science, 2006, 314, 1585-1588

[10] Yurke, B. and Mills, A. P. Using DNA to Power Nanostructures. Genetic Programming and Evolvable Machines, 2003, 4, 111-122 